\title{
Multi-Level Conditional Transit Signal Priority in Connected Vehicle Environments
}

\author{
Zorica Cvijovic ${ }^{\mathrm{a}}$, Milan Zlatkovic ${ }^{\mathrm{a}}$, Aleksandar Stevanovic ${ }^{\mathrm{b}}$, Yu Song $^{\mathrm{c}}$ \\ a University of Wyoming, Department of Civil and Architectural Engineering \\ ${ }^{b}$ University of Pittsburgh, Department of Civil and Environmental Engineering \\ c University of Wisconsin, Department of Civil and Environmental Engineering
}

\begin{tabular}{|c|c|}
\hline ARTICLE INFO & A B S TR A C T \\
\hline $\begin{array}{l}\text { DOI: 10.31075/PIS.67.02.01, } \\
\text { Professional paper } \\
\text { Received: 24/02/2021 } \\
\text { Accepted: 31/03/2021 } \\
\text { Corresponding author: } \\
\text { mzlatkov@uwyo.edu }\end{array}$ & \multirow{3}{*}{$\begin{array}{l}\text { Connected Vehicles (CV) are an emerging technology with a large potential to } \\
\text { improve traffic operations and safety. This paper develops and tests advanced } \\
\text { CV-based multi-level conditional Transit Signal Priority (TSP). The algorithms } \\
\text { are using the latitude/longitude (lat/lon) coordinates of CV vehicles and } \\
\text { intersections to establish communication, share information and request priority. } \\
\text { The TSP strategies are implemented through controllers' built-in features and } \\
\text { logic processor, using Econolite ASC/3 as a representative traffic signal } \\
\text { controller. The tests were performed in VISSIM microsimulation with the ASC/3 } \\
\text { Software-in-the-Loop (SIL) controller emulator. State Street in Salt Lake City, } \\
\text { UT, is selected as a test-case corridor. The paper shows that the developed } \\
\text { signal control priority (SCP) algorithms are successful in reducing delays for } \\
\text { target vehicles in excess of } 6 \% \text {, without significant impacts on other traffic. The } \\
\text { information obtained from CV vehicles can be used to further enhance control } \\
\text { algorithms and create adaptive SCP programs. }\end{array}$} \\
\hline Keywords: & \\
\hline $\begin{array}{l}\text { Connected Vehicles } \\
\text { Transit Signal Priority } \\
\text { Bus Rapid Transit } \\
\text { VISSIM } \\
\text { ASC/3 }\end{array}$ & \\
\hline
\end{tabular}

\section{Introduction}

Connected Vehicles (CV) are an emerging technology aim at improving safety and operations of transportation systems. CV technologies enable vehicles to exchange information with each other in real time using wireless-based communication technologies. The communication allows for sharing vital transportation information among the roadway users (ITSJPO). The CV equipment continually transmits vehicle position, direction, and speed, as well as other information (such as the status of vehicle's systems), to other vehicles in the vicinity. It also allows vehicles to communicate to equipment installed in the infrastructure, such as traffic signals, signs, work or school zones, railroad crossings and similar. The primary focus of the USDOT's CV program is in safety applications aimed at reducing crashes. However, these technologies have a significant potential in improving mobility and operations and reducing congestions (Yang, 2017). CV technologies are gaining a momentum in research and practice. The benefits of these technologies are just beginning to be recognized. The limited number of field tests have proven that they can be used for different adaptive traffic control programs. There are still many areas that need to be covered through research.

Special traffic signal operations, such as Transit Signal Priority (TSP) rely heavily on the communication between transit vehicles and the traffic signal controllers. Traditionally, this communication was achieved through traffic detectors (loops, video, radar), or wireless technologies, such as infrared, Global Positioning System (GPS) or radar. These technologies could mainly transmit the position of the vehicle and trigger a call for priority. CV technologies are a step up from this communication, since they can share a lot more information both ways between the approaching vehicles and the signal controllers, allowing for advanced and adaptive special signal operations. TSP facilitates the movement of transit vehicles, through signalized intersections by allowing for additional green time, early green, or other strategies. The benefits of TSP include reductions in bus delays, increase in operating speeds, improved schedule adherence, reduced bus bunching and increase in ridership, among others. However, it can have negative impacts on non-priority approaches and neighboring intersections (Park and Hu 2014). TSP is 
an essential element of surface rapid transit modes, such as Bus Rapid Transit (BRT).

The goal of this paper is to assess the benefits and impacts of a CV-based TSP control program applied to a congested urban corridor. The control programs were developed and tested in Econolite ASC/3 Software-in-the-Loop (SIL) signal control simulation implemented in VISSIM microsimulation software. The tests were performed on a 10-intersection corridor along State Street in Salt Lake City, UT, using 2015 traffic data and 2025 traffic projections. In addition to the built-in TSP features, this study also develops a 3level customized conditional TSP combined with a future BRT system along this corridor. The next section of the paper summarizes the major findings from the literature on $\mathrm{CV}$ technologies and special signal operations. It is followed by the description of the methodology applied in this research, results and discussion sections. The major conclusions of the paper are presented in the last section.

\section{Literature Review}

CV technologies enable vehicles to exchange information with each other and with the roadside infrastructure in real-time using wireless-based communication technologies. There are three base types of information exchange: vehicle-to-vehicle (V2V), vehicle-to-infrastructure or vice versa (V2I/I2V), and vehicle-to-device or vice versa (V2D/D2V). The last type includes vehicle-to-pedestrian (V2P) communication by cell phone devices, and vehicle-tocloud (V2C) information exchange (Johnson, 2017). Vehicle information that is being shared includes vehicle position, direction, and speed, as well as other information (such as the status of vehicle's systems), to the infrastructure and other vehicles in the vicinity. This information is known as the Basic Safety Message (BSM). BSM allows vehicles to communicate to equipment installed in the infrastructure, such as traffic signals, signs, work or school zones, railroad crossings and similar. The open-source protocol for wireless communication in $\mathrm{CV}$ applications is known as the dedicated short-range communication (DSRC), and is set by the Federal Communications Commission (FCC) for Intelligent Transportation Systems (ITS) (Narla and Stowell, 2019). DSRC transmits data 10 times per second within the range of about 1,000 ft (Glascock, 2016). Other wireless technologies, such as WiFi, WAVE, 3G/4G/5G, DMB can be used in CV applications. However, DSRC has an advantage by being more reliable, safe and secure (Yang, 2017; Glascock, 2016). The primary focus of the USDOT's CV program is in safety applications aimed at reducing crashes. However, these technologies have a significant potential in improving mobility and operations and reducing congestion (Yang et al., 2017). CV technologies are gaining a momentum in research and practice. The limited number of field tests have proven that they can be used for different adaptive traffic control programs.

TSP is an operational strategy that facilitates the movement of in-service transit vehicles through trafficsignal controlled intersections (Smith et al., 2005). It can be implemented as passive, active or adaptive. Passive TSP does not require detection, and is optimized based on the frequency of transit vehicles along a certain corridor. Active TSP is most common, and it requires detection of approaching transit vehicles. The detection can be achieved through loop, radar, video, infrared or GPS, among other means. Active TSP can be unconditional (where every transit vehicle requests and is granted priority), or conditional (where priority is granted only to certain transit vehicles, such as those running behind schedule, or carrying more passengers on board). Conditional TSP can also be headway-based or "Always-ON", where TSP will be granted to every transit vehicle during certain times of day or days of week (Kimley-Horn, 2017). Adaptive TSP weighs the benefits and impacts of transit and vehicular traffic when determining the priority given to transit vehicles. TSP is mostly achieved through green extension (GE) and/or early green (EG), where the transit vehicle receives extra green time at the end or beginning of the green phase, respectively. Other strategies include phase rotation (PR), phase insertion, separate transit phase and similar. The benefits of TSP include reductions in bus delays, increase in operating speeds, improved schedule adherence, reduced bus bunching and increase in ridership, among others. However, it can have negative impacts on non-priority approaches and neighboring intersections (Park and $\mathrm{Hu}, 2014)$. Several studies used field data to estimate the effectiveness of TSP. In Tacoma, Washington, TSP in combination with signal optimization reduced traffic signal delays around $40 \%$ in two corridors. Powell Boulevard bus line in Portland, Oregon recorded transit travel time improvements by up to $8 \%$ and a reduction in travel time variability by $19 \%$. In Chicago, the recorded reduction in bus running times was between $7 \%$ and $20 \%$, with a $44 \%$ reduction in bus intersection delays, depending on the time of day, which saved one bus weekly (TSP State-of-the-Practice, 2001). The bus travel time has reduced up to $25 \%$ in Los Angeles after TSP implementation (Smith et al., 2005).

As a more convenient approach, most studies have used simulation models to evaluate the effectiveness of the transit or freight signal priority, where fieldcollected data served to calibrate and validate the simulation models. An advantage of simulation studies is that they can develop and test new technologies in a virtual environment. Dion et al. (2005) used INTEGRATION microsimulation to evaluate TSP along transit corridors with different types of signals (Dion et al., 2004; Dion and Rakha, 2005). VISSIM microsimulation was applied by Chen et al. (2008), Ghanim et al. (2013), and Zlatkovic et al. (2013) in their evaluations of TSP on a BRT corridor in Beijing, a bus 
corridor in Michigan State University, and a BRT corridor in Salt Lake County, Utah, respectively. AlDeek et al. (2017) developed and simulated several scenarios to compare the effects of unconditional and conditional TSP coupled with both regular bus line and BRT. The simulation models were based on a 1.1-mile long I-Drive test-bed at Orlando, FL. The results revealed that unconditional TSP with and without BRT was the most effective in delay and travel time reduction. However, the models resulted in a significant side street delay increase, especially at intersections with high traffic demand. Therefore, the study proposed conditional TSP for BRT vehicles which were three minutes behind the schedule as the most beneficial solution, which would provide a balance between BRT benefits and impacts on other traffic (Al-Deek et al., 2017). A point/time detection or constant update of a vehicle location is of high importance for successful TSP. With the deployment of GPS-based detection, TSP performances improved significantly. However, it showed some shortages, such as the lack of accuracy or impossibility to support adequate sampling of vehicle locations for closely spaced intersections, or check-in/out calls if the midblock bus stop is close to the intersection. Further, urban environments may provide additional challenges for GPS-based systems (Smith et al., 2005). A theoretical model developed by Liu et al. (2007) identified the optimal time to place a call for transit priority operation for early green based on the predicted bus arrival time information. The bus arrival time was estimated using GPS technology. This theoretical approach was validated through a simulation model that aimed to identify the optimal calltime points for the general case. The final results showed that the TSP initiation with the bus about 2030 s away from the intersection can provide good results for both bus and other vehicular traffic either in moderately congested or congested traffic conditions (Liu et al., 2007). A study by Li et al. (2011) developed a model that was aiming to implement adaptive TSP on an actuated dual-ring traffic control system. The model was illustrated using a numerical example under medium and heavily congested traffic conditions. This adaptive TSP optimization used green splits for three consecutive cycles to minimize the weighted sum of transit vehicle delays and other traffic. A field implementation was built upon a distributed closedloop signal control system where controllers received calls or actuation from inductive loop detectors. The results showed that the average bus delay and average traffic delay along the bus movement direction, at a congested intersection, were reduced by approximately $43 \%$ and $16 \%$ respectively, while the average delay of cross-street traffic was increased by about $12 \%$ (Li et al., 2011). Using AIMSUN simulation models, Liao and Davis (2007) developed adaptive GPS-based TSP that could consider the bus schedule adherence, number of passengers on board, location, and speed. The communication between buses and signal controllers was assumed to be carried out through a wireless technology (either an already developed local area network or DSRC). Simulation results indicated that a $12 \%$ to $15 \%$ reduction in bus travel times during the morning, and a $4 \%$ to $11 \%$ reduction in the afternoon peak hour could be achieved by providing signal priority for buses. Due to the coordination along the corridor, the priority could not be allowed in each cycle (Liao and Davis, 2007). A study by Song et al. (2017) evaluated the effectiveness of conditional GPS-based TSP through microsimulation. The results showed that conditional GPS-based TSP can reduce transit travel times by up to $13 \%$, with a minimal negative impact on the sidestreet traffic (3\% delay increase) (Song et al., 2017).

A limited number of studies evaluated the effectiveness of CV systems for TSP implementation. CV technologies provide additional information on transit vehicles, such as speeds, arrival rates, position, acceleration, deceleration, stopped time and the number of the passenger on board, among other information contained in the BSM. Regardless of the type of communication, National Transportation Communication for ITS Protocol (NTCIP) standards are followed, hence TSP operates in the context of a "system" that includes a priority requester, such as a transit vehicle, traffic signal controllers, and the management centers that configure and monitor the traffic signal controllers. It, therefore, imposes functional and communications requirements on all of these "system" components. However, the availability of $\mathrm{CV}$ technologies for this purpose depends on the market penetration rate of $\mathrm{CV}$-equipped transit vehicles. A recent field implementation and study of CV-equipped buses along the Redwood Road corridor in Salt Lake County, UT, showed that the equipped buses that requested priority met their schedules $2 \%$ $6 \%$ more frequently than other buses. This field test also proved the reliability of that DSRC communication (Leonard et al., 2019). Other recent studies used traffic simulation to evaluate TSP effects in a CV environment. A study by Park and $\mathrm{Hu}$ (Park and $\mathrm{Hu}$, 2014) developed a TSP logic that takes advantage of CV technologies, including two-way communication between the bus and the traffic signal controller, bus location detection and prediction, the number of passengers on board, to allow an accurate reallocation of the green time. Microsimulation was used to compare the developed TSP against scenarios with conventional TSP and without TSP. The results showed a reduction in bus delay from $9 \%$ to $84 \%$ compared to conventional TSP, and from $36 \%$ to $88 \%$ compared to no-TSP. A study by Head et al. used VISSIM microsimulation to estimate the benefits of the Multimodal Intelligent (MMITS) in CV environment on two networks, in San Mateo CA and Maricopa County, AZ (University of Arizona, 2016). The study implemented MMITS strategies through ASC/3 SIL. The results showed a reduction in transit travel times of $8 \%-10 \%$ (San Mateo) and $11 \%-15 \%$ (Maricopa), as 
well as a reduction in transit delays of $23 \%-25 \%$ (San Mateo) and $32 \%-43 \%$ (Maricopa). A study by Anh et al. (2016) used the same approach (VISSIM with ASC/3 SIL) to assess the potential effects of a broader MMITSS deployment. For TSP implementation with CVs, the results showed that travel time reduced for both transit and passenger vehicles by up to $29 \%$ and $28 \%$, respectively. However, the study also found negative effects of TSP on system-wide delay because of the reduction of green times on side streets.

The study presented in this paper first tests the TSP features that exist in the traffic controllers. Then the study develops a 3-level custom conditional TSP for BRT implementation (no TSP, low TSP and high TSP) depending on the schedule adherence and the number of passengers on board using $\mathrm{CV}$ communication. The communication is based on the real-time latitude/longitude (lat/lon) coordinates of approaching vehicles and intersections. The methodologies developed in this paper are a first step toward creating CV-adaptive signal priority control programs, which will be explored in future research.

\section{Test-Case Corridor and Data Collection}

The test-case network for this study consists of a 10intersection corridor along State Street in Salt Lake City, UT, between 500 S street on the north and 2100 S street on the south end of the corridor, as shown in Figure 1. This is a multi-modal corridor that carries a significant amount of traffic, with AADT of close to 36,000 vehicles per day along the busiest sections (UDOT AADT map 2014). $500 \mathrm{~S}$ and $600 \mathrm{~S}$ are one way streets, WB and EB respectively, carrying traffic to and from $\mathrm{l}-15$, which is about $1.6 \mathrm{~km}$ west of State Street. The busiest intersection in the test-case corridor is $2100 \mathrm{~S}$, used by more than 9,500 vehicles during the PM peak period (4:00 - 6:00 PM), which is the interval used in this study. $700 \mathrm{~S}$, Kensington Avenue and $1910 \mathrm{~S}$ are minor streets/signalized driveways with insignificant traffic, therefore they were not included in the analysis. State Street also carries significant transit ridership. The major bus route along the corridor is Route 200, which is one of the Utah Transit Authority (UTA's) routes with highest ridership. This bus route is planned for a BRT conversion in the future. State Street has approximately $7 \%$ of truck traffic in the traffic flow. Bicycle traffic is high, because of the flat topography and the vicinity of the downtown area. Significant pedestrian traffic also exists in the area.

The data collection was performed in 2015 for the purpose of a previous multimodal study for the State Street corridor, and was used in this paper. The data were obtained through field data collection, the Utah Department of Transportation (UDOT) and UTA's databases, and included traffic volumes/intersection turning movements, travel times, signalized intersection control parameters and transit operation characteristics. These data are used to develop, calibrate and validate the base microsimulation model. The first dataset consisted of intersection turning movement counts, which included vehicular traffic, pedestrians and bicyclists. These data were collected in the field for seven signalized intersections within the analysis networks (excluding $700 \mathrm{~S}$, Kensington Avenue and $1910 \mathrm{~S}$ ), for the PM peak period (4:00 PM - 6:00 PM). UDOT's Automated Traffic Signal Performance Measures (ATSPM) system was also used for checking the turning movement counts, approach volumes and signal timing data.

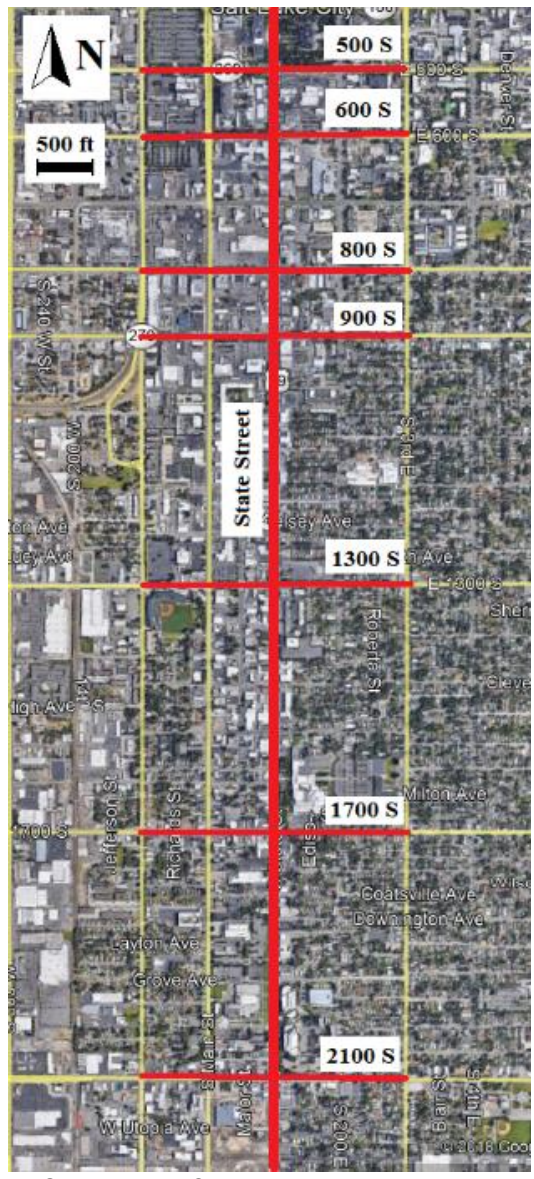

Figure 1. State Street Test Case Network Source: (GoogleEarth)

The second dataset consisted of travel time runs, which were performed using the floating car technique. Additional travel time data were obtained through online services, such as INRIX travel times, WAZE and Google Maps. The third dataset consisted of signal timing data, which were obtained from the UDOT Traffic Operations Center (TOC), in the form of pdf files for all ten signalized intersections in the analyzed network. The forth dataset consisted of transit data, including ridership, GPS, transit schedules and vehicle capacities. These data were obtained from UTA for September 2015 as the representative month for this analysis. All the collected data were used to develop the base microsimulation model. 


\section{Microsimulation Models}

The development, testing and assessment of the TSP algorithms were performed through VISSIM microsimulation, coupled with Econolite ASC/3 SIL controller software. Communication, BSM and Road Side Unit (RSU) operations were programmed in Python and embedded in microsimulation. All signal control operations, priority and controller logic were programmed directly in ASC/3 controllers. Five model scenarios were created as follows:

- Base model, which was created, calibrated and validated for 2015 PM traffic conditions.

- 2025 Do-Nothing model, which includes traffic projections for the year 2025 , using a $2.4 \%$ traffic growth rate obtained through the analysis of historical trends. The geometry of the model remained unchanged from the Base model, however traffic signals were optimized in Synchro for the projected demand levels.

- 2025 CV Transit Signal Priority (TSP) model, which introduced CV-equipped buses. It is assumed that all buses, as fleet vehicles, will be equipped with CV technologies by 2025 . This scenario, however, does not include CV-equipped passenger cars. A detailed description of the $\mathrm{CV}$ and TSP algorithms is provided in later subsections of the paper.

- 2025 BRT model, which introduces a centerrunning BRT line along State Street, with highercapacity buses (110 spaces per bus) running every 15 minutes, fewer bus stops located at highdemand locations with improved pedestrian access and increased ridership. The new ridership demand was computed based on the $2.4 \%$ yearly growth rate. The signals were re-phased for protected-only left turns because of the center BRT lanes, so Synchro was used to re-optimize the signal timings. Additional design and operational improvements included longer storage space for turn lanes at intersections and a dual left turn lane in the WB direction at the $2100 \mathrm{~S}$ intersection.

- 2025 CV BRT TSP model, which upgrades the previous model by introducing CV-equipped BRT vehicles and 3-level custom conditional TSP. A detailed description of the TSP algorithms is provided in later subsections of the paper.

Each model was created for the two-hour PM peak period (4:00 - 6:00 PM). The simulations included a 15-minute warm-up period and two hours of output recordings. The outputs were averaged from ten randomly-seeded simulation runs for each scenario, with the same sequence of random seeds among scenarios to allow for a reasonable comparison.

\subsection{Base Model}

The Base model was developed, calibrated and validated for 2015 existing traffic conditions along the State Street corridor. VISSIM version 11, coupled with Econolite ASC/3 SIL traffic controller software, was used for modeling. The data used in modeling include actual roadway and intersection geometries, traffic counts for vehicles, pedestrians and bicyclists, corridor travel times, transit route and other ridership data, and signal timing data. The outputs were averaged from ten simulation runs with different random seeds. The Base model was calibrated for intersection turning movements, and validated for corridor travel times. The calibration was performed for five major signalized intersections: 500 S, 600 S, 800 S, 1300 S and 2100 $\mathrm{S}$. For this purpose, the two-hour PM peak (4:00 6:00 PM) intersection turning movement counts were used. The comparison was performed for each approach and each movement separately. Figure 2. a) shows compiled calibration results for the five intersections and each movement, where the field counts were compared to the results obtained from the simulation. The coefficient of determination (R2) for calibration was close to 1.0 for the entire network, showing satisfactory calibration results. For validation purposes, the corridor was split into seven segments in each direction, where one segment was between a pair of major signalized intersections. Travel times collected in the field were used to validate the model. The field travel times were averaged from five travel time runs in each direction during the PM peak period, using the floating car technique. Model validation is shown in Figure 2. b) and 2. c). It represents a comparison between the average field travel times and the travel times obtained from the simulation, including the standard deviation. The R2 value for validation was 0.904 in the northbound (NB) and 0.961 in the southbound (SB) direction. The calibration and validation results show a good fidelity of the Base model.

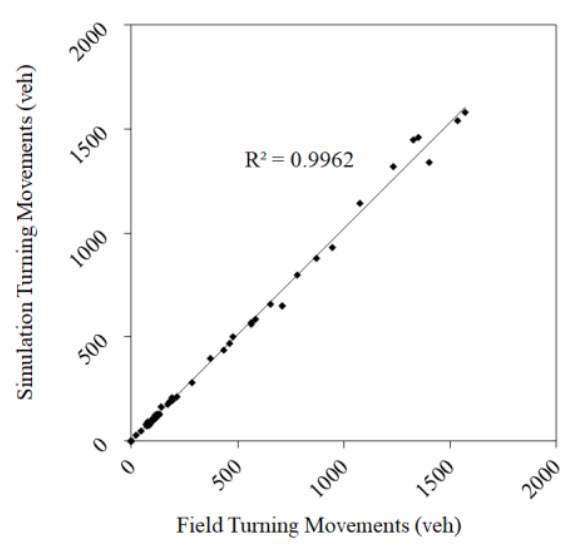

Figure 2 a). Base Model Calibration 


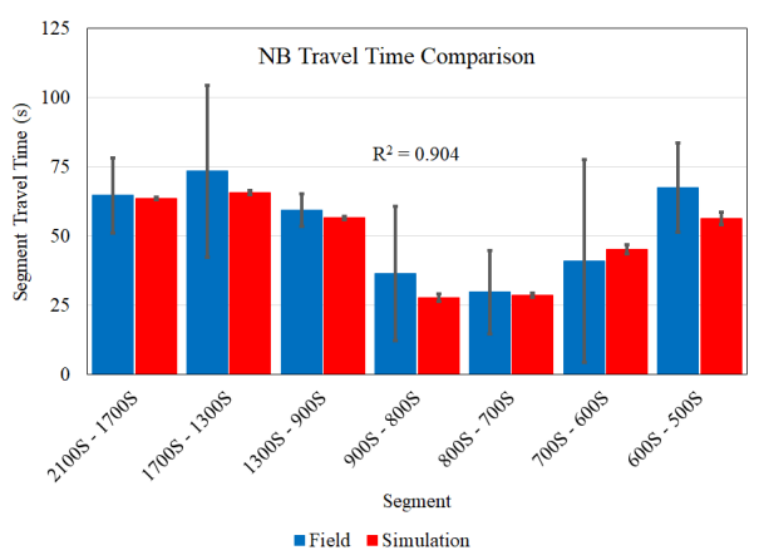

Figure 2 b). Base Model Validation NB

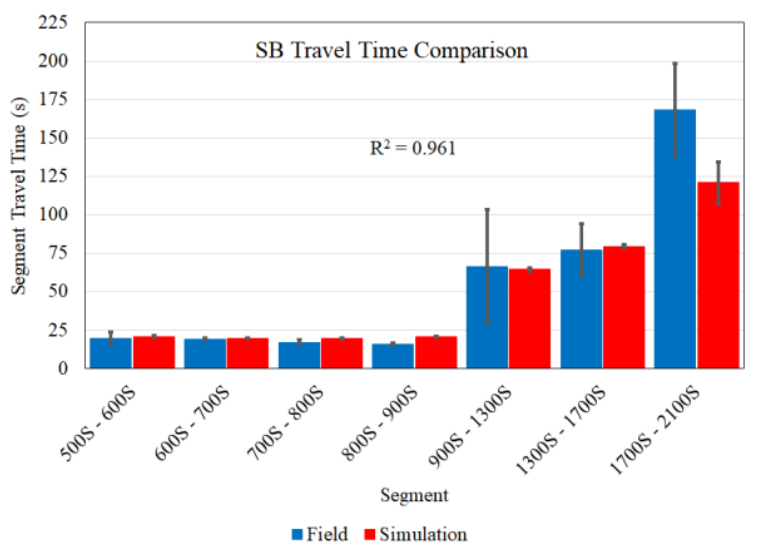

Figure 2 c). Base Model Validation SB

\subsection{CV Transit Signal Priority Model}

The 2025 Do-Nothing model was developed assuming $2.4 \%$ traffic growth in the area and did not include any geometrical modifications. Traffic signal timings were optimized through Synchro for the new demand levels. The 2025 CV TSP model was based on the 2025 DoNothing model, and introduced CVs and SCP for transit buses. No other vehicles were equipped with CV technologies in this study. The algorithm for 2025 CV TSP assumes that all (100\%) of buses will be CVequipped. When the transit vehicle enters the defined detection range, it starts sharing its location with the RSU. The distance between the bus and the intersection, as well as the estimation of the bus arrival time, are updated in each time step. According to the current signal phase and vehicle location, the RSU triggers TSP actuation. Each transit vehicle in the detection range is eligible for priority, regardless of the schedule adherence or bus capacity utilization, i.e. the priority is unconditional. When the vehicle leaves the intersection, the TSP is canceled. Following the constant updates of the distance between the bus and the intersection, the RSU can cancel TSP if it estimates that the bus cannot reach the intersection during the pre-defined time period. A new TSP initiation is possible in the next cycle.

The CV protocols, communication, BSM information and RSU operations were programmed through
VISSIM's Component Object Model (COM) using Python programming language. TSP operations were programmed through built-in signal control priority functions in $\mathrm{ASC} / 3$ controllers. The main information contained in the BSM that was used for communication with traffic signals through RSU was the vehicles' lat/lon coordinates. RSU contained the lat/lon coordinates of the center of the intersection, which was used as a reference point. As a vehicle was approaching the intersection, it would send its lat/lon coordinates, and the RSU would compute the current distance in every time step. The distance was computed using the Haversine formula as follows (GIS Map):

$$
\begin{aligned}
& \mathrm{a}=\sin ^{2}\left(\frac{\Delta \mathrm{lat}}{2}\right)+\cos \left(\mathrm{lat}_{\mathrm{veh}}\right) \cdot \cos \left(\mathrm{lat}_{\mathrm{int}}\right) \cdot \sin ^{2}\left(\frac{\Delta \mathrm{lon}}{2}\right) \\
& \mathrm{c}=2 \cdot \operatorname{atan} 2 \cdot(\sqrt{\mathrm{a}}, \sqrt{(1-\mathrm{a})}) \\
& \mathrm{d}=\mathrm{R} \cdot \mathrm{c}
\end{aligned}
$$

\section{Where:}

latveh, latint - latitude values of the vehicle and the center of the intersection, respectively

lonveh, lonint - longitude values of the vehicle and the center of the intersection, respectively

$\Delta$ lat - latveh - latint (difference of latitudes)

$\Delta$ lon $=$ lonveh - lonint $_{\text {(difference of longitudes) }}$

$\mathrm{R}$ - Earth radius $(6,371,000 \mathrm{~m}=20,902,231 \mathrm{ft})$

$\mathrm{d}$ - distance between the vehicle and the center of the intersection $(\mathrm{ft})$, updated every time step

The lat/lon coordinates in VISSIM were computed through user defined attributes which converted the current VISSIM into the world coordinates. The model was created on top of the world map provided as a background in VISSIM, which ensured correct lat/lon coordinates of the network. This process was repeated for each intersection in the network. Therefore, in each time step the intersection had the information about the position of the approaching vehicles. Although this procedure is developed in simulation, it can easily be transferred to field implementation, since lat/lon coordinates are contained in the BSM, while the RSU can have that information for the intersection.

The communication range between the vehicles and the intersections was set to $183 \mathrm{~m}(600 \mathrm{ft})$, mainly for two reasons. First, the intersections on the north side of the network are spaced at around $213 \mathrm{~m} \mathrm{(700} \mathrm{ft),} \mathrm{so}$ a longer communication range would contain two intersections at certain times. This was not optimal for the TSP requests in this case. Second, the green extension times were set to 15 seconds maximum, so the distance traveled by the vehicles at the speed limit (50 km/h =30 mph) was close to $198 \mathrm{~m}$ (650 ft) during 
that time, which would ensure an effective GE strategy.

Once a CV-equipped vehicle entered the $183 \mathrm{~m}$ (600 $\mathrm{ft}$ ) detection zone, it would be allowed to send a TSP request to the signal. In this study, SCP was provided only to the buses along the main corridor (NB and SB through on State Street), although the controller was able to identify those vehicles on all approaches. The vehicles on NB/SB through phases were filtered out based on the heading/route information from the BSM. The TSP request and strategies were programmed through a combination of RSU operations, and built-in controller logic and SCP features. When the RSU noticed a request, it would activate the internal controller logic and enable GE/EG TSP strategies. GE was set to 15 seconds maximum, while the maximum green time reductions for EG were set as 5 seconds for left turns along the main corridor, and 10 seconds for through movements from side streets. In this case, the TSP was unconditional, meaning that all CVs (all buses) would send a request and receive priority. The re-service cycle was set to one. The TSP check-out

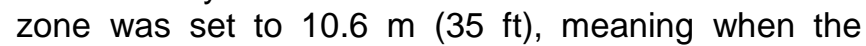
vehicle is within $10.6 \mathrm{~m}$ from the center of the intersection the call would be canceled. This was optimized for the current intersection geometries in this network.

\subsection{CV BRT TSP Model}

Because of the significant multimodal features of the State Street corridor, the local transportation agencies recognized the need for BRT implementation (The Planning Center 2011; UDOT 2015; Wasatch Front Regional Council 2015). This study considered a BRT implementation with center-running exclusive lanes, upgraded buses with 110 spaces capacity, upgraded stations and passenger routing. The BRT ridership demand was computed based on the existing data and travel demand projections. Signal operations were changed because of the center-running buses, therefore permitted left turns were not allowed along State Street. Synchro was used to optimize signal timings in this case. The 2025 BRT model included BRT operations, but did not incorporate TSP for comparison purposes. Due to the negative effects on the side-streets, BRT with unconditional TSP has not been considered for this study (Al-Deek et al. 2017). Furthermore, it is assumed that for the future BRT operations UDOT and UTA will implement conditional CV-based TSP, according to the current field tests (Leonard et al., 2019). The 2025 CV BRT TSP model included BRT operations, CV-equipped buses and 3level custom conditional TSP strategies. The CV communication, RSU operations, check-in and checkout actuation were the same as in the previously described CV model. The three levels of conditional TSP were defined as follows:
- No TSP: buses are running on time, and the BRT bus capacity utilization is less than $20 \%$

- Low TSP: buses are running on time, and the BRT capacity utilization is more than $20 \%$, OR buses are running $0-2$ minutes behind schedule, and bus capacity utilization is less than $20 \%$. GE/EG strategies are implemented in this case. GE was 15 seconds maximum, while the maximum green time reductions for EG were 5 seconds for left turns along the main corridor, and 10 seconds for through movements from side streets.

- High TSP: buses are running $0-2$ minutes behind schedule and the capacity utilization is more than $20 \%$, OR buses are running more than 2 minutes behind schedule, regardless of bus capacity utilization. This TSP includes GE/EG as previously described, with addition of phase rotation (PR). The signals along the main corridor operate with leading left phases in the regular mode. PR changes this sequence, allowing the stopped bus to leave the intersection earlier with leading through phases. PR works in combination with $E G$, while GE is provided to a vehicle that approaches the intersection during the green phase, if the TSP request is still active.

The BRT time-check points for each intersection were defined based on the free-flow speeds and station dwell times in both directions. These check points were used to assess the schedule adherence for each intersection. The $20 \%$ capacity utilization equals 22 passengers on board for the 110-space buses. These two parameters were used to determine the TSP level for each bus, direction and intersection. GE/EG strategies were programmed through built-in SCP in ASC/3 controllers. PR was achieved through the controllers' logic processor by activating an alternate action plan with a sequence that was programmed with leading through phases. The multi-level TSP algorithm is shown in Figure 3. 


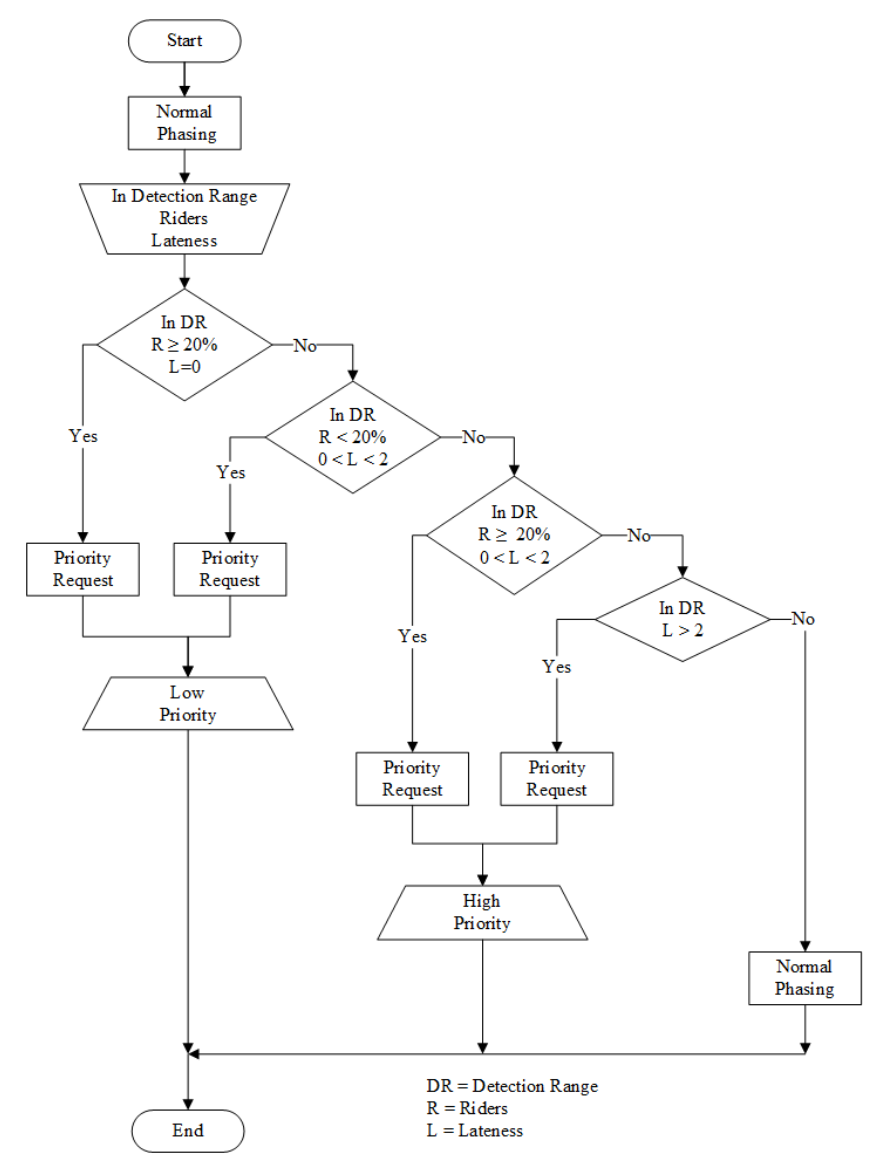

Figure 3. Three-Level Conditional TSP

\section{Results and Discussion}

The results collected from the simulations and used in the analysis include intersection performance measures, vehicle speeds, and phase split durations. The results were collected and averaged from ten simulation runs for each model. Where applicable, twotailed paired t-test with a 95\% confidence level was used to check if the differences in results were statistically significant.

\subsection{Intersection Performance}

Intersection performance for the seven major intersections on the corridor ( $500 \mathrm{~S}, 600 \mathrm{~S}, 800 \mathrm{~S}, 900$ S, $1300 \mathrm{~S}, 1700 \mathrm{~S}$ and $2100 \mathrm{~S}$ ) was assessed through the average vehicle delays per vehicle type for each scenario. The vehicle delays, weighted for the entire intersection, are provided in Table 1.

Table 1. Intersection Performance: Vehicle Delays

\begin{tabular}{|c|c|c|c|c|c|c|}
\hline \multirow{3}{*}{ Intersection } & \multirow{3}{*}{ Mode } & \multicolumn{5}{|c|}{ Average Delay per Vehicle (s) } \\
\cline { 3 - 7 } & & $\begin{array}{c}\mathbf{2 0 2 5} \\
\text { Do- } \\
\text { Nothing }\end{array}$ & $\begin{array}{c}\mathbf{2 0 2 5} \\
\text { CV } \\
\text { TSP }\end{array}$ & $\begin{array}{c}\mathbf{2 0 2 5} \\
\text { BRT }\end{array}$ & $\begin{array}{c}\mathbf{2 0 2 5} \\
\text { CV } \\
\text { BRT } \\
\text { TSP }\end{array}$ \\
\hline \multirow{3}{*}{$500 \mathrm{~S}$} & Car & 31.7 & 81.2 & 91.2 & 88.8 & 95.0 \\
\cline { 2 - 7 } & Bus & 52.6 & 95.3 & 102.9 & 19.2 & 26.3 \\
\cline { 2 - 7 } & Truck & 34.1 & 87.7 & 86.7 & 91.9 & 98.3 \\
\hline \multirow{3}{*}{$600 \mathrm{~S}$} & Car & 35.7 & 60.0 & 112.4 & 28.2 & 32.6 \\
\cline { 2 - 7 } & Bus & 15.6 & 53.7 & 117.3 & 54.1 & 46.9 \\
\cline { 2 - 7 } & Truck & 37.9 & 62.5 & 78.5 & 30.5 & 35.1 \\
\hline
\end{tabular}

\begin{tabular}{|c|c|c|c|c|c|c|}
\multirow{3}{*}{$800 \mathrm{~S}$} & Car & 33.7 & 42.7 & 47.9 & 38.1 & 38.9 \\
\cline { 2 - 7 } & Bus & 34.3 & 56.9 & 48.0 & 14.9 & 12.2 \\
\cline { 2 - 7 } & Truck & 35.7 & 44.9 & 57.3 & 42.5 & 42.7 \\
\hline \multirow{3}{*}{$900 \mathrm{~S}$} & Car & 13.9 & 21.4 & 21.0 & 20.1 & 22.6 \\
\cline { 2 - 7 } & Bus & 14.6 & 14.2 & 21.3 & 21.2 & 22.1 \\
\cline { 2 - 7 } & Truck & 16.5 & 21.9 & 12.0 & 22.1 & 25.5 \\
\hline \multirow{3}{*}{$1300 \mathrm{~S}$} & Car & 41.3 & 55.4 & 68.8 & 41.9 & 43.4 \\
\cline { 2 - 7 } & Bus & 19.6 & 47.1 & 56.0 & 30.9 & 30.2 \\
\cline { 2 - 7 } & Truck & 40.6 & 59.6 & 50.8 & 43.2 & 45.5 \\
\hline \multirow{3}{*}{$1700 \mathrm{~S}$} & Car & 14.9 & 62.6 & 84.0 & 38.9 & 27.8 \\
\cline { 2 - 7 } & Bus & 12.6 & 58.4 & 83.3 & 34.1 & 28.1 \\
\cline { 2 - 7 } & Truck & 16.4 & 64.6 & 56.2 & 40.7 & 30.0 \\
\hline \multirow{3}{*}{$2100 \mathrm{~S}$} & Car & 41.2 & 94.9 & 126.2 & 73.8 & 74.9 \\
\cline { 2 - 7 } & Bus & 37.8 & 81.0 & 127.9 & 35.8 & 31.6 \\
\cline { 2 - 7 } & Truck & 44.8 & 95.6 & 82.2 & 76.5 & 78.1 \\
\hline \multirow{3}{*}{ Average } & Car & $\mathbf{3 0 . 4}$ & $\mathbf{5 9 . 7}$ & $\mathbf{7 8 . 8}$ & $\mathbf{4 7 . 1}$ & $\mathbf{4 7 . 9}$ \\
\cline { 2 - 7 } & Bus & $\mathbf{2 6 . 7}$ & $\mathbf{5 8 . 1}$ & $\mathbf{7 9 . 5}$ & $\mathbf{3 0 . 0}$ & $\mathbf{2 8 . 2}$ \\
\cline { 2 - 7 } & Truck & $\mathbf{3 2 . 3}$ & $\mathbf{6 2 . 4}$ & $\mathbf{6 0 . 5}$ & $\mathbf{4 9 . 6}$ & $\mathbf{5 0 . 7}$ \\
\hline
\end{tabular}

The average vehicle delays would increase $50 \%$ or more for all vehicle types in 2025 if no improvements are made along the corridor. The $1700 \mathrm{~S}$ and $2100 \mathrm{~S}$ intersections would experience the most increase in vehicle delays, almost up to four times. All the increases are statistically significant ( $t$ test $=-7.623, p$ $=2.43 \mathrm{E}-07$ ). The unconditional TSP significantly increase delay for cars and buses, in excess of $35 \%$, while for the trucks, delay reduces $10 \%$ to $40 \%$ in this scenario. Bus priority is not effective in this case. Therefore, the unconditional TSP is not recommended for implementation for high percentage of target vehicles. BRT implementation without TSP benefits all modes, with the decrease in delays most evident for buses (close to $50 \%$ compared to 2025 Do-Nothing). Cars and trucks experience a $21 \%$ delay reduction. The implementation of the 3-level conditional TSP has different effects on an intersection-by-intersection basis. In general, it reduces BRT delays by $6 \%$ while it increases car and truck delays by up to $2 \%$. None of these changes is statistically significant (car: $t=-$ $0.357, p=0.733$; bus: $t=1.007, p=0.353 ; t=-0.529, p$ $=0.616)$. The increase in car and truck delays is mostly attributed to the left turns along State Street and through movements on side streets.

\subsection{Vehicle Speeds}

The vehicle speed results are given in Tables 2 - 4 . The speeds were measures in each direction for each vehicle type, on segments between the seven major intersections (14 segments in total). The 2025 DoNothing scenario reduces speeds for all vehicle types between $11 \%$ and $31 \%$, and all these reductions are statistically significant. The introduction of BRT operations improves speeds for all vehicle types, most evidently for transit, when compared to 2025 DoNothing. Car and truck speeds increase about $15 \%$, while transit speeds increase more than $70 \%$. The transit speed increase is statistically significant. The unconditional bus TSP generally reduces speeds for all vehicles when compared to 2025 Do-nothing. The $17 \%$ reduction in car speeds is statistically significant, while the bus and truck speed reductions are not. Due 
to the exclusive bus lanes and upgraded operations, transit speeds in the 2025 BRT scenario are higher than those in the Base scenario. The introduction of TSP does not have statistically significant impacts on any of the speeds (car: $t=0.768, p=0.456$; bus: $t$ $=0.469, p=0.646 ; t=0.546, p=0.594)$. It does improve transit speeds on majority of segments, but this increase is not significant. Car and truck speeds remain mainly unchanged.

Table 2. Car Speed Comparison

\begin{tabular}{|c|c|c|c|c|c|c|}
\hline \multicolumn{7}{|c|}{ Car Speeds $(\mathrm{km} / \mathrm{h})$} \\
\hline \multicolumn{2}{|r|}{ Segments } & Base & $\begin{array}{c}2025 \\
\text { DN }\end{array}$ & $\begin{array}{c}2025 \\
\text { CV TSP }\end{array}$ & $\begin{array}{l}2025 \\
\text { BRT }\end{array}$ & $\begin{array}{l}2025 \text { CV } \\
\text { BRT TSP }\end{array}$ \\
\hline \multirow{7}{*}{ SB } & $500 \mathrm{~S}-600 \mathrm{~S}$ & 41.68 & 19.31 & 18.99 & 21.24 & 18.35 \\
\hline & $600 \mathrm{~S}-700 \mathrm{~S}$ & 44.74 & 42.65 & 44.90 & 28.97 & 26.07 \\
\hline & $700 \mathrm{~S}-800 \mathrm{~S}$ & 45.06 & 17.22 & 16.25 & 17.54 & 17.22 \\
\hline & 800 S - 900 S & 41.84 & 32.03 & 32.83 & 37.98 & 33.80 \\
\hline & $900 S-1300 S$ & 50.86 & 25.11 & 22.21 & 52.30 & 50.05 \\
\hline & $1300 S-1700 S$ & 40.56 & 8.85 & 8.05 & 20.92 & 32.03 \\
\hline & $1700 S-2100 S$ & 26.39 & 7.24 & 7.40 & 9.98 & 12.55 \\
\hline \multirow{7}{*}{ NB } & $2100 S-1700 S$ & 50.86 & 34.44 & 33.31 & 35.41 & 35.89 \\
\hline & $1700 S-1300 S$ & 49.08 & 35.57 & 34.12 & 38.62 & 37.01 \\
\hline & $1300 S-900 S$ & 57.78 & 53.59 & 53.91 & 55.04 & 53.75 \\
\hline & $900 \mathrm{~S}-800 \mathrm{~S}$ & 31.06 & 33.47 & 27.36 & 36.53 & 34.28 \\
\hline & $800 \mathrm{~S}-700 \mathrm{~S}$ & 30.42 & 37.34 & 18.67 & 38.79 & 37.98 \\
\hline & $700 \mathrm{~S}-600 \mathrm{~S}$ & 19.15 & 15.45 & 7.08 & 22.05 & 16.25 \\
\hline & $600 \mathrm{~S}-500 \mathrm{~S}$ & 15.45 & 14.81 & 9.66 & 16.90 & 15.61 \\
\hline \multicolumn{2}{|r|}{ Average } & 38.92 & 26.93 & 23.91 & 30.88 & 30.06 \\
\hline
\end{tabular}

Table 3. Bus Speed Comparison

\begin{tabular}{|c|c|c|c|c|c|c|}
\hline \multicolumn{7}{|c|}{ Bus Speeds (km/h) } \\
\hline & Segments & Base & $\begin{array}{c}2025 \\
\text { DN }\end{array}$ & $\begin{array}{c}2025 \\
\text { CV TSP }\end{array}$ & $\begin{array}{l}2025 \\
\text { BRT }\end{array}$ & $\begin{array}{l}2025 \text { CV } \\
\text { BRT TSP }\end{array}$ \\
\hline \multirow{7}{*}{ SB } & $500 S-600 S$ & 22.85 & 10.30 & 12.23 & 16.09 & 17.22 \\
\hline & $600 S-700 S$ & 30.58 & 30.74 & 30.42 & 12.23 & 14.00 \\
\hline & $700 \mathrm{~S}-800 \mathrm{~S}$ & 15.13 & 10.94 & 11.10 & 41.36 & 37.82 \\
\hline & $800 S-900 S$ & 18.67 & 16.42 & 16.09 & 48.12 & 39.43 \\
\hline & $900 S-1300 S$ & 25.27 & 16.90 & 14.81 & 22.53 & 21.08 \\
\hline & $1300 S-1700 S$ & 29.77 & 7.72 & 7.24 & 21.40 & 23.01 \\
\hline & $1700 S-2100 S$ & 18.67 & 6.60 & 6.76 & 23.34 & 24.46 \\
\hline \multirow{7}{*}{ NB } & $2100 S-1700 S$ & 24.46 & 23.50 & 24.30 & 23.17 & 24.94 \\
\hline & $1700 S-1300 S$ & 29.93 & 23.82 & 24.46 & 25.75 & 27.52 \\
\hline & $1300 S-900 S$ & 29.13 & 29.45 & 29.77 & 19.63 & 19.63 \\
\hline & $900 \mathrm{~S}-800 \mathrm{~S}$ & 22.69 & 13.04 & 13.20 & 49.41 & 50.21 \\
\hline & $800 S-700 S$ & 16.42 & 17.70 & 11.75 & 55.20 & 55.04 \\
\hline & $700 S-600 S$ & 15.61 & 9.98 & 6.44 & 10.14 & 10.14 \\
\hline & $600 S-500 S$ & 10.14 & 8.53 & 8.53 & 20.44 & 19.31 \\
\hline \multicolumn{2}{|r|}{ Average } & 22.09 & 16.12 & 15.51 & 27.77 & 27.42 \\
\hline
\end{tabular}

Table 4. Truck Speed Comparison

\begin{tabular}{|c|c|c|c|c|c|c|}
\hline \multicolumn{7}{|c|}{ Truck Speeds (km/h) } \\
\hline \multicolumn{2}{|r|}{ Segments } & Base & $\begin{array}{c}2025 \\
\text { DN }\end{array}$ & $\begin{array}{c}2025 \\
\text { CV TSP }\end{array}$ & $\begin{array}{l}2025 \\
\text { BRT }\end{array}$ & $\begin{array}{c}2025 \\
\text { CV BRT } \\
\text { TSP }\end{array}$ \\
\hline \multirow{7}{*}{ SB } & $500 S-600 S$ & 40.88 & 18.67 & 18.51 & 20.92 & 17.38 \\
\hline & $600 S-700 S$ & 45.06 & 42.97 & 46.51 & 29.13 & 28.00 \\
\hline & $700 S-800 S$ & 45.71 & 17.22 & 15.45 & 16.90 & 16.90 \\
\hline & $800 S-900 S$ & 39.75 & 31.06 & 32.99 & 35.89 & 30.26 \\
\hline & $900 S-1300 S$ & 47.15 & 22.85 & 20.76 & 42.81 & 44.42 \\
\hline & $1300 S-1700 S$ & 41.36 & 8.69 & 8.05 & 20.92 & 29.13 \\
\hline & $1700 S-2100 S$ & 25.75 & 7.24 & 7.40 & 10.14 & 12.23 \\
\hline \multirow{7}{*}{ NB } & $2100 S-1700 S$ & 50.69 & 33.80 & 32.19 & 35.41 & 35.24 \\
\hline & $1700 S-1300 S$ & 43.94 & 31.87 & 32.99 & 35.57 & 31.70 \\
\hline & $1300 S-900 S$ & 48.60 & 50.05 & 47.80 & 51.82 & 50.86 \\
\hline & $900 S-800 S$ & 26.07 & 25.59 & 23.50 & 29.13 & 31.87 \\
\hline & $800 S-700 S$ & 33.80 & 37.66 & 18.67 & 39.11 & 38.30 \\
\hline & $700 S-600 S$ & 17.70 & 16.09 & 7.08 & 18.67 & 15.93 \\
\hline & $600 S-500 S$ & 15.61 & 13.84 & 9.50 & 18.51 & 15.61 \\
\hline \multicolumn{2}{|r|}{ Average } & 37.29 & 25.54 & 22.96 & 28.92 & 28.42 \\
\hline
\end{tabular}

\subsection{Phase Split Durations}

Phase split durations are provided in Table 5 for each of the seven major intersections. Only splits for through phases are shown, since the left turn phases can be skipped, depending on the left turn demand. Most split durations in the 2025 Do-Nothing scenario are statistically significantly different than the corresponding splits in the Base scenario. This is to be expected, since the signals were optimized for 2025 demand. For the same reason the split durations in both BRT scenarios are statistically significantly different than the corresponding splits in the 2025 DoNothing scenario. Split durations for majority of intersections in the 2025 CV TSP scenario are significantly different than the corresponding ones in 2025 Do-Nothing. This is due to the redistribution of green times caused by GE/EG for phases 2 and 6 . Differences can also be seen between 2025 BRT and 2025 CV BRT TSP scenarios, however they are not statistically significant in most cases. An interesting observation can be made for phases 2 and 6 in those two scenarios. These phase splits are in most cases shorter in the BRT TSP scenario, regardless of the $\mathrm{GE} / \mathrm{EG}$ strategies. This can be attributed to the coordination, since the duration of these phases would be changed to maintain coordination between intersections. Furthermore, major adjustments caused by coordination had to be made when PR was active. However, no significant differences were observed. 
Table 5. Phase Split Duration

\begin{tabular}{|c|c|c|c|c|c|c|}
\hline \multirow[b]{2}{*}{ Node } & \multirow[b]{2}{*}{ Phase } & \multicolumn{5}{|c|}{ Phase Splits (s) } \\
\hline & & Base & $\begin{array}{c}2025 \\
\text { DN }\end{array}$ & $\begin{array}{c}2025 \mathrm{CV} \\
\text { TSP }\end{array}$ & $\begin{array}{l}2025 \\
\text { BRT }\end{array}$ & $\begin{array}{l}2025 \mathrm{CV} \\
\text { BRT TSP }\end{array}$ \\
\hline \multirow{3}{*}{$500 \mathrm{~S}$} & 2 & 62.5 & 61.5 & 69.2 & 70.6 & 63.3 \\
\hline & 6 & 50.8 & 46.1 & 53.6 & 49.7 & 42.1 \\
\hline & 8 & 52.0 & 53.0 & 56.7 & 43.9 & 44.6 \\
\hline \multirow{3}{*}{$600 \mathrm{~S}$} & 2 & 71.8 & 54.7 & 56.5 & 45.7 & 37.3 \\
\hline & 4 & 42.4 & 55.2 & 62.7 & 50.6 & 54.9 \\
\hline & 6 & 72.0 & 59.4 & 63.1 & 63.9 & 53.6 \\
\hline \multirow{4}{*}{$800 \mathrm{~S}$} & 2 & 72.8 & 63.3 & 68.2 & 57.3 & 57.3 \\
\hline & 4 & 33.0 & 37.3 & 41.8 & 31.2 & 31.4 \\
\hline & 6 & 71.7 & 65.1 & 69.6 & 57.0 & 56.6 \\
\hline & 8 & 38.1 & 41.4 & 46.4 & 35.5 & 35.8 \\
\hline \multirow{4}{*}{$900 \mathrm{~S}$} & 2 & 84.8 & 70.7 & 73.4 & 61.1 & 60.4 \\
\hline & 4 & 27.6 & 42.7 & 50.1 & 43.6 & 45.2 \\
\hline & 6 & 85.9 & 70.8 & 74.7 & 66.6 & 65.9 \\
\hline & 8 & 27.6 & 42.7 & 50.1 & 43.6 & 45.2 \\
\hline \multirow{4}{*}{$1300 \mathrm{~S}$} & 2 & 74.3 & 68.8 & 72.3 & 56.6 & 54.2 \\
\hline & 4 & 34.1 & 36.9 & 42.1 & 32.9 & 36.3 \\
\hline & 6 & 74.8 & 64.0 & 68.1 & 55.7 & 53.3 \\
\hline & 8 & 38.1 & 42.6 & 46.9 & 36.4 & 39.5 \\
\hline \multirow{4}{*}{$1700 \mathrm{~S}$} & 2 & 83.0 & 73.8 & 74.9 & 75.2 & 75.7 \\
\hline & 4 & 24.8 & 34.0 & 38.2 & 25.8 & 25.8 \\
\hline & 6 & 82.6 & 69.1 & 70.8 & 67.1 & 67.6 \\
\hline & 8 & 31.9 & 40.9 & 49.0 & 33.5 & 33.3 \\
\hline \multirow{4}{*}{$2100 \mathrm{~S}$} & 2 & 60.3 & 55.8 & 60.0 & 53.0 & 50.4 \\
\hline & 4 & 35.5 & 35.2 & 40.5 & 33.7 & 33.3 \\
\hline & 6 & 46.0 & 44.0 & 52.1 & 46.6 & 45.9 \\
\hline & 8 & 46.4 & 43.6 & 45.9 & 37.8 & 37.2 \\
\hline
\end{tabular}

\section{Conclusions}

The goal of this paper was to develop, test and assess the effectiveness of conditional TSP in various CV applications in a congested urban corridor. The control programs were developed and tested in Econolite ASC/3 SIL signal control simulation implemented in VISSIM microsimulation. CV protocols, communication, BSM information and RSU operations were implemented through Python programming language. TSP operations were programmed through built-in SCP functions in ASC/3 controllers. The algorithms used lat/lon coordinates of intersections and approaching vehicles to determine the detection zone. The study developed a 3-level customized conditional TSP that uses bus schedule adherence and real-time ridership to determine the level of TSP for each approaching vehicle (no TSP, low TSP or high TSP).The tests were performed on a 10-intersection corridor along State Street in Salt Lake City, UT.
The analysis of the test-case corridor shows a major deterioration of future traffic conditions if no improvements are made. Vehicle delays would increase and speeds reduce significantly, about 50\% and $30 \%$ respectively, with a significant deterioration in transit operations. The implementation of unconditional TSP for buses provided significant delay savings for trucks, up to $40 \%$, but it also caused significant delay increase for other vehicles, in excess of $35 \%$. Speeds for all vehicles would reduce if unconditional TSP is provided to all target vehicles. A BRT implementation would benefit all transportation modes, reducing delays by more than $20 \%$ and improving speeds by $15 \%$ for general-purpose traffic. Transit delays would reduce more than $50 \%$ and their speeds increase more than $70 \%$.

The information that is sent from CV-equipped transit vehicles can be used to create different forms of conditional priority. This study used schedule adherence and real-time ridership to determine the level of granted TSP for BRT. Green extension, early green and phase rotation were the strategies implemented for different combinations of vehicle lateness and ridership. The introduced TSP strategies generally reduced transit delays by about $6 \%$, without significant impacts on other traffic and transit operations.

This study is the first step toward creating field-ready traffic control programs for special signal operations. For this study, only the vehicles' position, heading, schedule adherence and ridership were used to develop signal control priority algorithms. However, CV-equipped vehicles can provide a lot more information that can be used to further fine tune traffic control. Furthermore, it only assumed that transit vehicles will be fully equipped. In reality, it can be expected that a percentage of other vehicles will also have CV equipment. Future studies will be focused on expanding the conditional priority algorithms with additional constraints, as well as developing adaptive SCP programs that use the information from all CVequipped vehicles, as well as real-time data from other traffic sensors and systems, such as the ATSPM.

\section{Acknowledgements}

The authors thank UDOT and UTA personnel for furnishing the data and their help with initiating the study. This study was partially supported by the Wyoming Department of Transportation (contract No. RS03219), and the Mountain Plains Consortium (MPC) University Transportation Center. The work presented herein remains the sole responsibility of the authors. 


\section{References}

[1] A Joint Standard of AASHTO, ITE, and NEMA. National Transportation Communication for ITS Protocol (NTCIP 1211 v02). 2014. Object Definitions for Signal Control and Prioritization (SCP)

[2] Ahn, K., Rakha, H. A., Kang, K., and Vadakpat, G. 2016. "Multimodal Intelligent Traffic Signal System Simulation Model Development and Assessment." Transportation Research Record, Journal of the Transportation Research Board. Volume 2558: 92-102. DOI: 10.3141/2558-09

[3] Al-Deek, H., Sandt, A., Alomari, A., and Hussain, O. (2017). "A technical note on evaluating the effectiveness of bus rapid transit with transit signal priority." Journal of Intelligent Transportation Systems, Taylor \& Francis, 21(3), 227-238.

[4] Chen, X., Yu, L., Zhu, L., Yu, L., and Guo, J. 2008. "Microscopic Simulation Approach to Effectiveness Analysis of Transit Signal Priority for Bus Rapid Transit: A Case Study in Beijing." Transportation Research Record. Volume 2072: 64-76.

[5] Dion, F., Rakha, H., and Zhang, Y. 2004 "Evaluation of Potential Transit Signal Priority Benefits along a Fixed-Time Signalized Arterial". Journal of Transportation Engineering. 130(3): 294-303.

[6] Dion, F. and Rakha, H. 2005. "Integration of Transit Signal Priority within Adaptive Traffic Signal Control Systems". Presented at 84th Annual Meeting of the Transportation Research Board, Washington, D.C.

[7] Ghanim, M.S., Abu-Lebdeh, G., and Ahmed, K. 2013. "Microscopic Simulation Study of Transit Signal Priority Implementation along an Arterial Corridor." Presented at 5th International Conference on Modeling, Simulation and Applied Optimization (ICMSAO), Hammamet, Tunisia.

[8] GIS Map: Haversine Formula. www.igismap.com/haversine-formula-calculategeographic-distance-earth/. Assessed May 23, 2019.

[9] Glascock, S. 2016. Connected and Autonomous Vehicles (CAV) Technology Team. Louisiana Department of Transportation and Development.

[10] Intelligent Transportation Systems Joint Program Office (ITSJPO). n. d. "Connected Vehicles." Accessed Sep 21, 2018. www.its.dot.gov/cv_basics.

[11] Johnson, C. 2017. Readiness of the road network for connected and autonomous vehicles. The Royal Automobile Club Foundation for Motoring Ltd., London, UK.
[12] Kimley-Horn. 2017. Concept of Operations and Systems Engineering Management Plan: Implementation of TSP on Murfreesboro Pike Corridor. Nashville

[13] Leonard, B., Mackey, J., Sheffield, M., Basset, D., Larson, S., and Hooper, I. 2019. "Demonstrating Transit Schedule Benefits with a Dedicated ShortRange Communication-Based Connected Vehicle System." Transportation Research Record, Journal of the Transportation Research Board. 110. DOI: 10.1177/0361198119859321

[14] Li, M., Yin, Y., Zhang, W., Zhou, K., and Nakamura, H. (2011). "Modeling and implementation of adaptive transit signal priority on actuated control systems." Computer-Aided Civil and Infrastructure Engineering, Wiley Online Library, 26(4), 270-284.

[15] Liao, C.-F., and Davis, G. A. (2007). "Simulation study of bus signal priority strategy: taking advantage of global positioning system, automated vehicle location system, and wireless communications." Transportation research record, SAGE Publications Sage CA: Los Angeles, CA, 2034(1), 82-91.

[16] Liu, H., Lin, W.-H., and Tan, C. (2007). "Operational strategy for advanced vehicle location system-based transit signal priority." Journal of Transportation Engineering, American Society of Civil Engineers, 133(9), 513-522.

[17] Narla, S. R. K., and Stowell, H. G. 2019. " Convergence of the Automotive, Telecommunications, Software, and Transportation Industries, and the Transportation Professional's Role." ITE Journal, 89(3): 28-33.

[18] Park, B and Hu, J. 2014. Transit Signal Priority with Connected Vehicle Technology. Mid-Atlantic Universities Transportation Center Report UVA2012-04, University of Virginia.

[19] Smith, H.R., Hemily, B. and Ivanovic, M. 2005. Transit Signal Priority (TSP): A Planning and Implementation Handbook, ITS America.

[20] Song, Y., Zlatkovic, M., and Porter, R.J. 2017. "Evaluation of GPS-based Transit Signal Priority for Mixed-Traffic Bus Rapid Transit." Transportation Research Record, Journal of the Transportation Research Board. Volume 2539: 30-39

[21] The Planning Center. Life on State: Our Street, Our Vision. Salt Lake City, UT, 2011.

[22] TSP State-of-the-Practice, Regional Transit Signal Priority Location Study-Phase II, Model Simulation. Innovative Transportation Concepts, Inc., Regional Transportation Authority, Chicago, IL, 2001. 
[23] University of Arizona, University of California PATH, Savari Networks Inc., and Econolite. MultiModal Intelligent Traffic Signal System Phase II: System Development, Deployment and Field Test. Connected Vehicle Pooled Fund Study, 2016.

[24] Utah Department of Transportation. Utah's Unified Transportation Plan 2011 - 2040. Salt Lake City, UT, 2015.

[25] Utah Department of Transportation. 2014 AADT map. Assessed July 20, 2019.

[26] http://uplan.maps.arcgis.com/home/webmap/view er.html?webmap=cf41e8473956413f906cfc96172 fe998.

[27] Wasatch Front Regional Council. Regional Transportation Plan 2015 - 2040. Salt Lake City, UT, 2015.

[28] Yang, C.Y.D., Ozbay, K. and Ban, X. 2017. "Developments in connected and automated vehicles." Journal of Intelligent Transportation Systems. 21(4): 251-254. DOI: 10.1080/15472450.2017.1337974

[29] Yang, X. 2017. "Traffic Operations with Connected and Automated Vehicles." Presented at AAAl-17 Al-CAV Workshop, San Francisco, CA

[30] Zlatkovic, M., Stevanovic, A., and Reza, R.Z. 2013. "Effects of Queue Jumpers and Transit Signal Priority on Bus Rapid Transit." Presented at 92nd Annual Meeting of the Transportation Research Board, Washington D.C. 